Communications in Physics, Vol. 20, No. 2 (2010), pp. 143-150

\title{
EFFECT OF EXCITATION MIGRATION AND UPCONVERSION IN HIGHLY ERBIUM-DOPED GLASS MICRO-SPHERE LASERS
}

\author{
PHAM VAN HOI \\ Institute of Materials Science, VAST \\ and \\ University of Engineering and Technology \\ BUI HUY \\ Institute of Materials Science, VAST \\ DO THUY CHI \\ Thai Nguyen University of Education
}

\begin{abstract}
Excitation migration and upconversion process in the Erbium-doped silica-alumina glass microsphere lasers with concentrations of 2500-4000 ppm were investigated in detail. The experiment shows that under $976 \mathrm{~nm}$ excitation, the intense of up-conversion emission at 523, 546 and $657 \mathrm{~nm}$, corresponding to the transitions ${ }^{2} H_{11 / 2} \rightarrow{ }^{4} I_{15 /},{ }^{4} S_{3 / 2} \rightarrow{ }^{4} I_{15 / 2}$, and ${ }^{4} F_{9 / 2} \rightarrow{ }^{4} I_{15 / 2}$, respectively, depends on the erbium content, migration of excitation and pump power. The excitation migration has strongly influenced on the threshold and Red shift of lasing wavelength and migration-assisted up-conversion process leads to degraded amplification performance of microsphere lasers made by silica-alumina glasses with different contents of Er-ions.
\end{abstract}

\section{INTRODUCTION}

Recently, the investigations of up-conversion processes have attracted great interest in connection with the development of Erbium-doped glasses with high doping concentration of Erbium ions $\left(10^{20}-10^{21} \mathrm{~cm}^{-3}\right)$ for planar amplifiers, and micro-cavity lasers operating at $1550 \mathrm{~nm}$ [1-3]. In other hand, the conversion of infrared light to visible light through energy up-conversion in rare-earth doped glasses, due to the possibility of infrared-pumped visible lasers, has a great potential application in areas such as optical data storage, lasers, sensors, and optical displays [4-6]. The increase of Erbium concentration leads to degraded amplifier performance owing to migration-assisted up-conversion processes [7-8]. To characterize the performance of a high -concentration optical amplifiers, model accounting for the up-conversion of excitation on homogeneously distributed (homogeneous up-conversion, or HUC) and clustered erbium ions (pair-induced quenching, or PIQ) have been used. The earlier models describing the up-conversion in Er-doped fiber assumed that the migration is so mush faster than the up-conversion that it smoothes out the excitation distribution even though all the ions are randomly distributed. This approximation resulted in a linear dependence of the up-conversion rate on the population inversion. Philipsen and Bjarklev proposed the Monte-Carlo technique for investigation of the up-conversion phenomenon in [9], that for randomly distributed ions, the up-conversion 
rate is a non-linear function of the population inversion and that it is accelerated by migration. In this description, the ensemble of all the excited ions was divided into two sub-ensembles: equidistant isolated ions and the clustered ones. However, a detailed microscopic study of Erbium-doped glasses by means of X-ray absorption fine structure spectroscopy has found no evidence of short-range pair clustering of Erbium ions [10]. Therefore, more accurate physical models have to be used for fitting experimental results [11].

In this work we experimentally investigate the excitation migration and up-conversion processes in the very high-concentration Erbium-doped silica-alumina glasses in the form of fiber and microphere bulk. The experiment shows that under $976 \mathrm{~nm}$ laser diode (LD) excitation, the intense of up-conversion emission at 523, 546 and $657 \mathrm{~nm}$, corresponding to the transitions ${ }^{2} \mathrm{H}_{11 / 2} \rightarrow{ }^{4} \mathrm{I}_{15 /},{ }^{4} \mathrm{~S}_{3 / 2} \rightarrow{ }^{4} \mathrm{I}_{15 / 2}$, and ${ }^{4} \mathrm{~F}_{9 / 2} \rightarrow{ }^{4} \mathrm{I}_{15 / 2}$, respectively, depends on the Erbium content, migration of excitation and pump power. The excitation migration and up-conversion process in the highly Er-doped glasses were verified by increase of threshold, Red shift of laser emission wavelength at the threshold and non-linear decrease of lasing power of microsphere lasers based on silica-alumina glasses with different contents of Er-ions.

\section{BACKGROUND}

\section{II.1. Micro- and macroscopic characterization of excitation migration and up- conversion process}

Migration of excitation and up-conversion process in the frame of microscopic characterization are based on excited state absorption (ESA) and the non-radiative excitation transition in pairs of optical centers. According to microscopic characterization of migration and up-conversion processes in [12], the rate of conversion transition is inversely proportional to the six power of distance between ions and the oscillator strength of corresponding transitions from ground and excited states.

The macroscopic approach consists in the application of rate equations to populations of energy levels and the temporal variation of number density for a metastable level is usually described by following equation:

$$
\frac{\partial N_{2}}{\partial t}=-A N_{2}+B u(\nu) N_{1}-C_{u p} N_{2}^{2}
$$

Here, the first term is the number of spontaneously emitted photons, the second term is the number of pumping transitions from ground state, and the third term is the number of up-conversion transition, $N_{1}$ and $N_{2}$ are number densities for ground and metastable levels, $A$ is the rate of spontaneous emission, $u(\nu)$ is the density of pumping radiation, $B$ is the Einstein coefficient for absorption. It is usually supposed that the number of up-conversion transition is proportional to the square of the population at metastable level, $N_{2}$, because two excited atoms are needed for up-conversion transition. The proportionality coefficient, $C_{u p}$, is considered as a macroscopic quantitative characteristic of the up-conversion process. In general, the $C_{u p}$ parameter is a function of time and may depend on the concentration of Er-ions, pumping and time recording of luminescence, when a pulse excitation is used. In an experiment, the researcher always measures 
macroscopic parameters, which are results of the averaging of individual pair properties and up-conversion coefficient, $C_{u p}$, is constant.

\section{II.2. Experiments}

In our experiments, we have used $\mathrm{SiO}_{2}: \mathrm{Al}_{2} \mathrm{O}_{3}$ (silica-alumina) glasses doped with $\mathrm{Er}$ and Y/Er with Er-concentration varied from 2500 ppm to $4000 \mathrm{ppm}$ (Table 1). The Erdoped silica-alumina glasses with a fiber form have been used for ensuring sufficient pump power density into the doping area. The Er-doped fiber was a commercial silica-alumina fiber with a typical core/cladding diameter ratio of 9/125 $\mu \mathrm{m}$ and Er-doped area diameter of $3 \mu \mathrm{m}$. The sol-gel glass samples with the composition (mol \%) $90 \mathrm{SiO}_{2}-6 \mathrm{Al}_{2} \mathrm{O}_{3}: 4 \mathrm{Y}_{2} \mathrm{O}_{3}$ : $\mathrm{xEr}_{2} \mathrm{O}_{3}$, where $\mathrm{x}=0.125$ and/or 0.2 (it is equivalent to $0.25-0.4 \mathrm{~mol} \%$ of $\mathrm{Er}^{3+}{ }_{\text {-ion }}$ in glass matrix). The Er-sample bulk has a spherical form with diameter varied from 90 to $120 \mu \mathrm{m}$. The method for making multi-component and micro-sphere Er-doped glasses was shown in our previous work [13]. We choose $976 \mathrm{~nm}$ - laser diode with adjustable output power in the range from 0 to $170 \mathrm{~mW}$ in single-mode emission (SDLO-2564-170) for excitation of Erbium ions dispersed into the both kinds of samples. In the case of spherical samples, we had used two different half-taper optical fibers for the pumping and the collecting light emission from the samples. The taper fibers were fabricated by chemical etching a standard single-mode fiber. The waist diameters of half-taper fiber were from 1 to $4 \mu \mathrm{m}$, which may be optimized to phase matching and coupling to the fundamental Whispering Gallery Modes (WGMs). The spectra of infrared light were measured by Optical Spectrum Analyzer (OSA) Advantest Q8384 made in Japan of $0.01 \mathrm{~nm}$ resolution and the up-conversion spectra were analyzed by monochromator MicroSpec 2300i, silicon CCD camera with resolution of $0.1 \mathrm{~nm}$ and sensitivity of $-90 \mathrm{dBm}$.

Table 1. Concentration of $\mathrm{Er}^{3+}$ and $\mathrm{Y}^{3+}$ ions

\begin{tabular}{lcccc}
\hline \hline \multirow{2}{*}{ \# Sample } & \multirow{2}{*}{ Glass } & \multirow{2}{*}{ Sample form } & \multicolumn{2}{c}{ Concentration $(\mathrm{ppm})$} \\
\cline { 4 - 5 } & & & $\mathrm{Er}^{3+}$ & $\mathrm{Y}^{3+}$ \\
\hline \hline 1 & Silica-alumina & Fiber & 2500 & - \\
2 & Silica-alumina & Fiber & 4000 & - \\
3 & Silica-alumina & Micro-Sphere & 4000 & - \\
4 & Silica-alumina (Solgel) & Micro-Sphere & 2500 & 80000 \\
5 & Silica-alumina (Solgel) & Micro-Sphere & 4000 & 80000 \\
\hline \hline
\end{tabular}

\section{RESULTS AND DISCUSSIONS}

Figure 1 demonstrates a simplified energy level diagram of the visible up-conversion emissions from the $\mathrm{Er}^{3+}$-ions in the glass matrix of silica-alumina by a $976 \mathrm{~nm}$ laser diode excitation. The $\mathrm{Er}^{3+}$-ion was populated on ${ }^{4} \mathrm{I}_{11 / 2}$ level through the ground state absorption by this scheme: ${ }^{4} \mathrm{I}_{15 / 2}+$ pumped photon (p-photon) $\rightarrow{ }^{4} \mathrm{I}_{11 / 2}$. Then the excited state absorption (ESA) occurred by two ions at ${ }^{4} \mathrm{I}_{11 / 2}$ populated one $\mathrm{Er}^{3+}$ ion to the ${ }^{4} \mathrm{~F}_{7 / 2}$ level and other one to ground state $\left({ }^{4} \mathrm{I}_{11 / 2}(\mathrm{~A})+{ }^{4} \mathrm{I}_{11 / 2}(\mathrm{~B}) \rightarrow{ }^{4} \mathrm{I}_{15 / 2}(\mathrm{~A})+{ }^{4} \mathrm{~F}_{7 / 2}(\mathrm{~B})\right)$, and a nonradiative decay from ${ }^{4} \mathrm{~F}_{7 / 2}$ to ${ }^{2} \mathrm{H}_{11 / 2},{ }^{4} \mathrm{~S}_{3 / 2}$, and ${ }^{4} \mathrm{~F}_{9 / 2}$ levels. Finally, the green 
and red up-conversion emissions centered at about 523, 546 and $660 \mathrm{~nm}$ were produced by the emitting transition of ${ }^{2} \mathrm{H}_{11 / 2} \rightarrow{ }^{4} \mathrm{I}_{15 / 2},{ }^{4} \mathrm{~S}_{3 / 2} \rightarrow{ }^{4} \mathrm{I}_{15 / 2}$ and ${ }^{4} \mathrm{~F}_{9 / 2} \rightarrow{ }^{4} \mathrm{I}_{15 / 2}$ of $\mathrm{Er}^{3+}$, respectively.

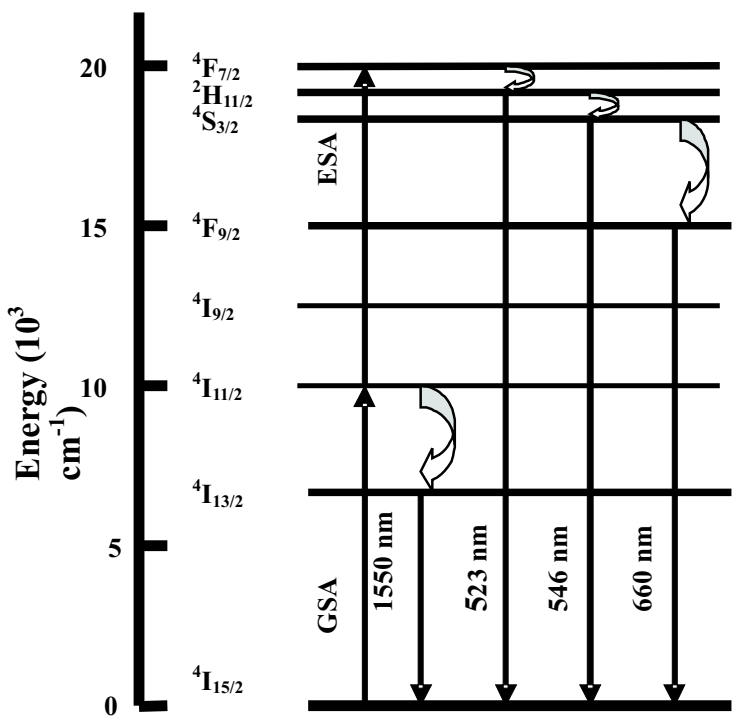

Fig. 1. Energy level diagram of green and red up-conversion emissions from the $\mathrm{Er}^{3+}$ ions distributed into $\mathrm{SiO}_{2}: \mathrm{Al}_{2} \mathrm{O}_{3}$ glasses by a $976 \mathrm{~nm}$ laser diode excitation.

Figure 2a shows the spectra of up-conversion emissions in the wavelength range of $500-670 \mathrm{~nm}$ for the $\mathrm{Er}^{3+}$ ions in the silica-alumina glass matrix excited by $976 \mathrm{~nm}$ laser diode. The green up-conversion emission spectra exhibited two green emission bands (figure $2 \mathrm{~b}$ ) centered at about 523 and $546 \mathrm{~nm}$, which were attributed to the ${ }^{2} \mathrm{H}_{11 / 2} \rightarrow{ }^{4} \mathrm{I}_{15 /}$, and ${ }^{4} \mathrm{~S}_{3 / 2} \rightarrow{ }^{4} \mathrm{I}_{15 / 2}$ of $\mathrm{Er}^{3+}$ ions in the silica-alumina glasses, respectively. The energy gap between the ${ }^{2} \mathrm{H}_{11 / 2}$ and ${ }^{4} \mathrm{~S}_{3 / 2}$ levels (in the range of $760-900 \mathrm{~cm}^{-1}$ depending on the host glass matrix) could be obtained from the green up-conversion emission spectra. At the quasithermal equilibrium at room temperature the fluorescence intensity of the transitions of ${ }^{4} \mathrm{~S}_{3 / 2} \rightarrow{ }^{4} \mathrm{I}_{15 / 2}$ (at wavelength of $546 \mathrm{~nm}$ ) is more than of ${ }^{2} \mathrm{H}_{11 / 2} \rightarrow{ }^{4} \mathrm{I}_{15 / 2}$ (at $523 \mathrm{~nm}$ ). In general, the fluorescence intensity ratio (FIR) of the two emissions varied by temperature [14].

Figure 3 shows a plot of the up-conversion emission intensity at wavelength peaks of 523 and $546 \mathrm{~nm}$ as a function of pumping power of $976 \mathrm{~nm}$ laser diode. This experimental result is good evidence to support for the Monte-Carlo technique proposed by Philipsen and Bjarklev in [9] for investigation of the up-conversion phenomenon, that the up-conversion rate is a non-linear function of the population inversion and that it is accelerated by migration of excitation. This result is promising for making green light laser from erbiumdoped glasses.

To verify the migration of excitation and up-conversion process in the highly Erdoped silica-alumina glasses, we investigated the lasing characteristics of micro-sphere lasers based on silica-alumina glasses doped $2500 \mathrm{ppm}$ and/or $4000 \mathrm{ppm}$ of $\mathrm{Er}^{3+}$ ions. We 


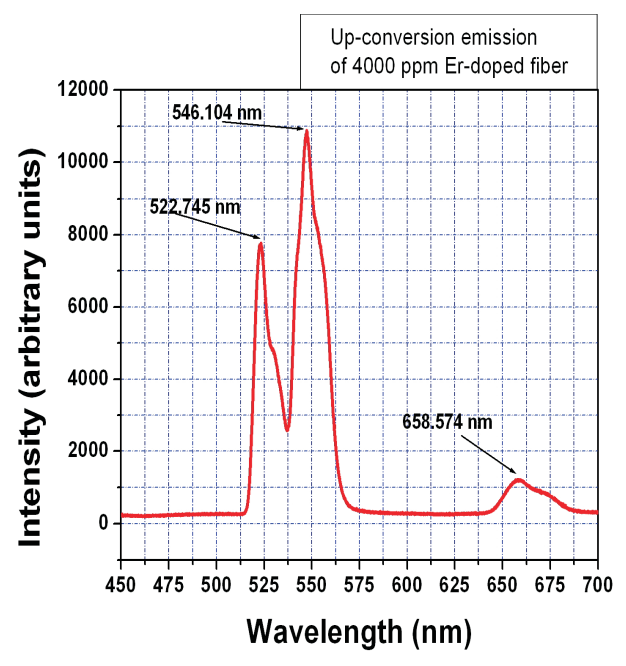

(a)

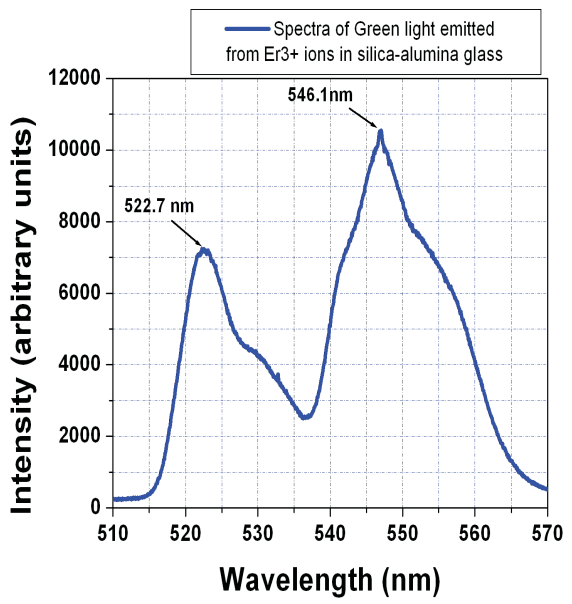

(b)

Fig. 2. Up-converion emission spectra in the wavelength range of 500- $600 \mathrm{~nm}$ (a) and green light emissions (b) for the $\mathrm{Er}^{3+}$-doped silica-alumina glasses with 4000 ppm-concentration of Er-ions

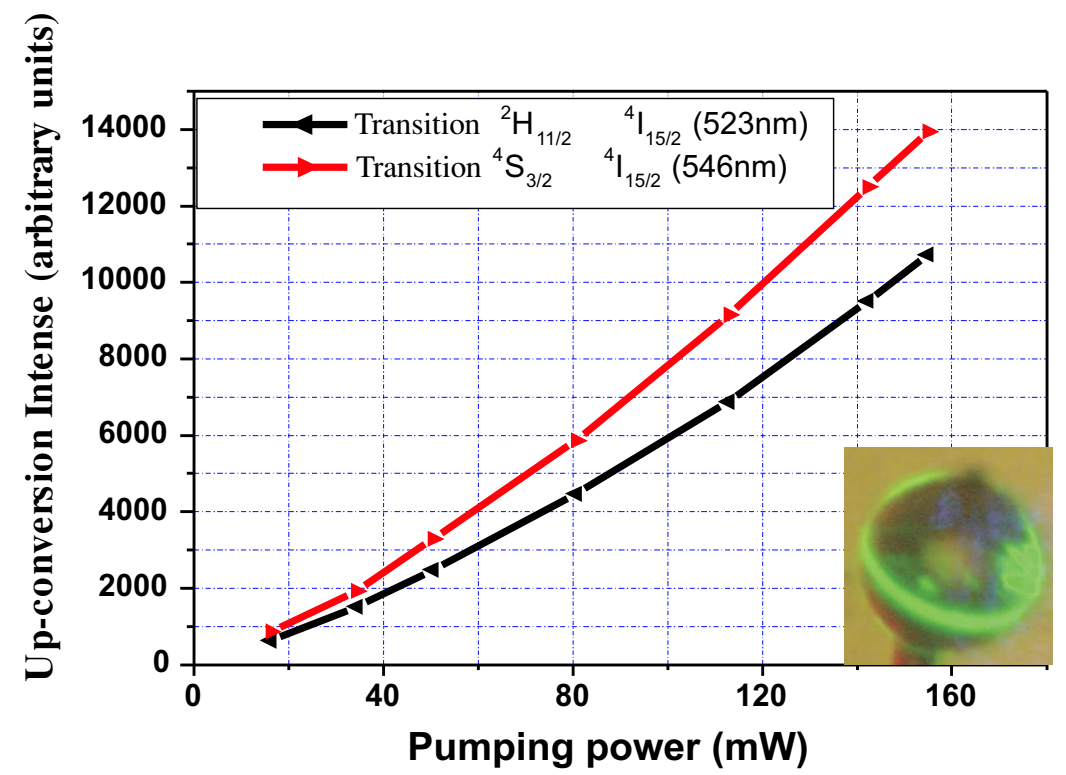

Fig. 3. Non-linearly dependence of Green up-conversion emissions at 523 and 546 $\mathrm{nm}$ versus pumping power of $976 \mathrm{~nm}$ laser diode. Inset: Image of upconversion green light emission from microsphere laser.

had obtained good results of the WGM emissions from sol-gel Er-doped glass micro-sphere 
lasers in our previous works $[13,15]$. The optimal ratio of $\mathrm{Al}_{2} \mathrm{O}_{3}: \mathrm{Er}_{2} \mathrm{O}_{3}$ and optimal $\mathrm{Er}^{3+}$ concentration dispersed into sol-gel silica-alumina glasses for micro-sphere lasers were 10:1 and of $2500 \mathrm{ppm}$, respectively. In comparison with previous studies, we have made the micro-sphere lasers with diameters of 90-120 $\mu \mathrm{m}$ based on Er-doped silica-alumina glass of $4000 \mathrm{ppm}$ of $\mathrm{Er}^{3+}$-ions concentration. Figure 4 demonstrates the supper-luminescence emissions from $2500 \mathrm{ppm}$ and $4000 \mathrm{ppm}$ Er-doped micro-sphere lasers with the pumped power below the threshold of $1.5 \mathrm{~mW}$ and of $2.5 \mathrm{~mW}$ at $976 \mathrm{~nm}$ LD excitation, respectively. We can observe the laser oscillation modes (WGMs) of varied Er-doped silica-alumina microsphere lasers with a diameter of $90-120 \mu \mathrm{m}$, when the optical pump power at $976 \mathrm{~nm}$ was below the laser threshold. The WGM laser oscillations were in the large wavelength range from $1510 \mathrm{~nm}$ to $1610 \mathrm{~nm}$, which was in the both C-band and L-band for telecom. Here the Er-ion concentration was ranging $0.25-0.4 \mathrm{~mol}_{\%}$ of $\mathrm{Er}_{2}^{3+}$. It is remarkable that with increasing Er- ion concentration from $2500 \mathrm{ppm}$ to $4000 \mathrm{ppm}$ the amplitude of supper-luminescence emissions would be non-linearly decreased (from - $60 \mathrm{dBm}$ to -76 $\mathrm{dBm}$ in our cases).

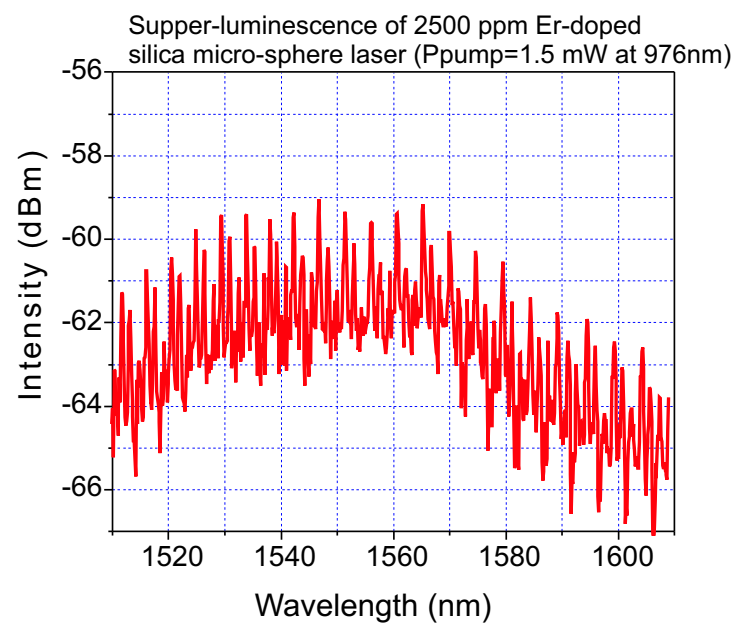

(a)

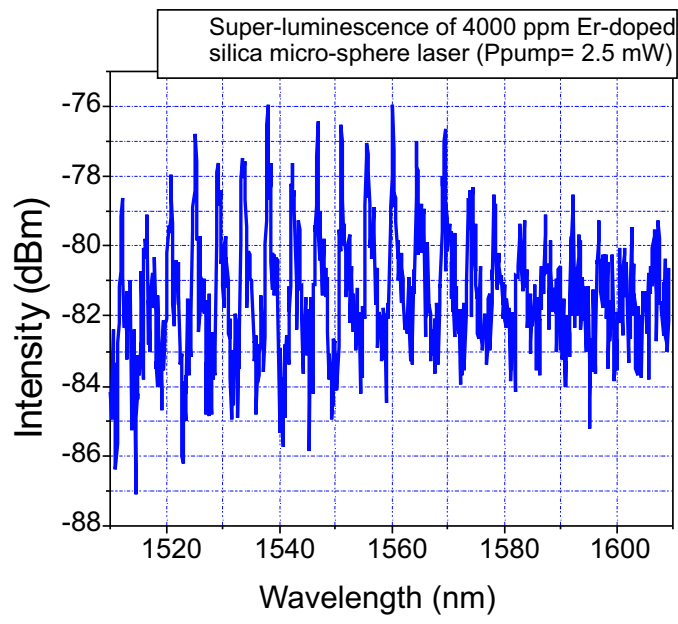

(b)

Fig. 4. Supper-luminescence emissions measured from micro-cavity lasers with diameter of $110 \mu \mathrm{m}$ under 976nm LD excitation. a) SL emission from $2500 \mathrm{ppm}$ and b) from 4000 ppm Er-doped silica-alumina glasses

Figure 5 shows the single-mode lasing spectra of WGM from microsphere lasers with Er-ion content changed from $2500 \mathrm{ppm}$ to $4000 \mathrm{ppm}$. In the case of homogeneous bulk $2500 \mathrm{ppm}$ Er-doped glass laser, the threshold was of $2 \mathrm{~mW}$ and the lasing wavelength peak was at about $1600 \mathrm{~nm}$. For the $2500 \mathrm{ppm}$ Er-doped glass-coated laser, the threshold was increased to the $2.5 \mathrm{~mW}$ and the wavelength peak was shifted to the 1557 nm. The peak intensity of output power at laser threshold of both cases was of $-55 \mathrm{dBm}$ (Fig. 5a). Microsphere laser based on 4000 ppm Er-doped silica-alumina glass has very 


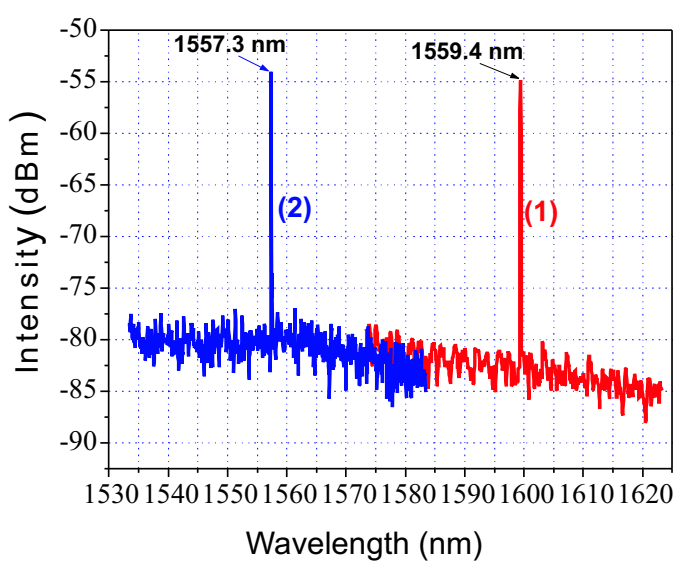

(a)

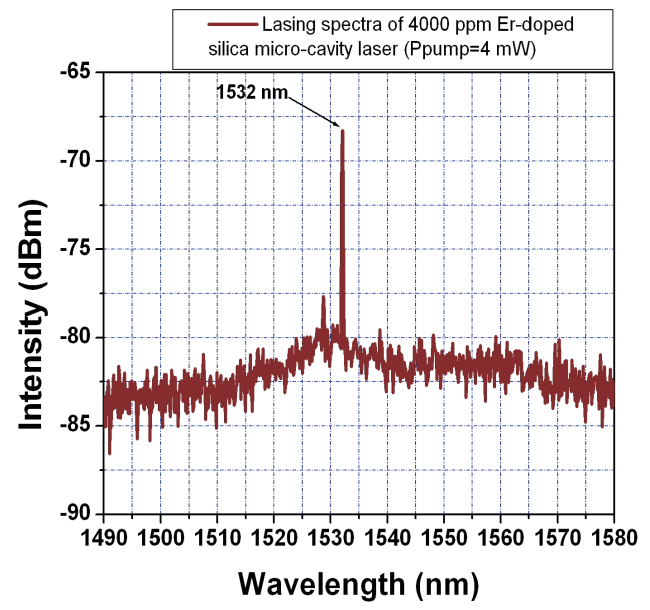

(b)

Fig. 5. a) Single-WGM spectra of solgel 2500 ppm Er-doped glass micro-sphere lasers at threshold $\left(\mathrm{P}_{\text {pump }} \approx 2-2.5 \mathrm{~mW}\right):(1)$ solgel bulk glass microsphere and (2) solgel coated glass microsphere. b) Single-WGM spectra of $4000 \mathrm{ppm}$ bulk Er-doped glass micro-sphere at threshold pump of $4 \mathrm{~mW}$. Diameter of sphere was of $110 \mu \mathrm{m}$ for all cases.

high threshold, and in some case the threshold would be increased for two times in comparison with $2500 \mathrm{ppm}$ Er-doped glass lasers and the lasing wavelength peak was shifted to the $1532 \mathrm{~nm}$ (Fig. 5b). The lasing power peak at threshold of $4000 \mathrm{ppm}$ Er-doped glass laser was of $-68 \mathrm{dBm}$, which decreased on $13 \mathrm{~dB}$ (equivalent to 20 times less) in comparison with $2500 \mathrm{ppm}$ Er-doped glass lasers. The obtained results of decreasing light emission intensity of IR-range with increasing Er-ion concentration can be explained by [16]: (i) the higher the surrounding density is, the faster is the up-conversion, that means the rate of up-conversion would be proportional to the concentration of excited ions.(ii) Since the up-conversion is fastest in the regions of the highest Er-ion density, it causes holes in the excitation distribution and migration efficiently fills up those holes by bringing excitations from the lower-density regions, where the up-conversion would be with a lower rate. Hence, the up-conversion is accelerated by migration. In our experiments, the migration of excitation may be directly influenced on the emission spectra of the Er-doped glasses and the migration-assisted up-conversion process leads to degraded amplification performance in the IR-range.

\section{CONCLUSIONS}

In summary, we report new experimental results of the up-conversion emissions in the highly $\mathrm{Er}^{3+}$-doped silica-alumina glasses of the form of fiber and spherical bulk. The intensity and emitting wavelength range of visible and IR emissions were investigated in detail based on spectrum characteristics of luminescence and lasing emissions from Er-doped glass micro-cavity lasers. The up-conversion rate is a non-linear function of 
the population inversion and that it is accelerated by migration of excitation. The IR emission intense had been decreased on 13-16 dB and the lasing wavelength at threshold was shifted to the red-wavelength range, when the $\mathrm{Er}^{3+}$ - ion concentration was increased from $2500 \mathrm{ppm}$ to $4000 \mathrm{ppm}$ in micro-cavity Er-doped glass lasers. The obtained results are good evidence of phenomenon of migration-assisted up-conversion process in the highly $\mathrm{Er}^{3+}$-doped silica-alumina glasses.

\section{ACKNOWLEDGEMENTS}

This work is financially supported by the State Key S\&T Program on Information and Communication Technology (Grant No. KC.01.06/06-10) and by the Basics Research Program of MOET (Grant No.B2008-TN04-06). The authors acknowledge Prof. Acad. Nguyen Van Hieu for his helpful discussions and support in this research.

\section{REFERENCES}

[1] B. C. Hwang, S. Jiang, T. Luo, K. Seneschal, G. Sorbello, M. Morell, F.Smektala, S.Honkanen, J. Lucas, N. Peyghambarian, IEEE Photon. Technol. Lett. 13 (2001) 197-199

[2] S. Tammela, M. Hotoleanu, P. Kiiveri, H. Valkonen, S. Sarkilahti, and K. Janka, Proc. Conf. On Optical Fiber Communications, v.1 of 2003 OSA Technical Digest Series (Opt. Soc. of America, 2003) (2003) 376-377

[3] P. Myslinski, D. Nguyen, and J. Chrotowski, J. Lightwave Technol. 15 (1997) 112-119

[4] O.Toma, S. Georgescu, IEEE J. Quantum Electron. 42 (2006) 192-197

[5] E. Heuman, S. Bar, K. Rademaker, G. Huber, S. Butterworth, A.Diening, and W. Seelert, Appl. Phys. Lett. 88 (2006) , 061108

[6] M. Pollnau, W. Luthy, and H. P. Weber, J. Appl. Phys. 77, No.12 (1995) , 6128-6134

[7] C Jiang, W. Hu, Q. Zeng, IEEE J.Quantum Electron. 39 (2003) 1266-1271

[8] S. Sergeyev, S. Popov, and A. T. Friberg, Opt. Lett. 30 (2005) 1258-1260

[9] J. L. Philipsen, A. Bjarklev, IEEE J. Quantum Electron. 35 (1999) 1741

[10] P. M. Peter, S. N. Houde-Walter, J. Non-Cryst. Solids, 328 (1998) 161-169

[11] S. Sergeyev, S. Popov, D. Khoptyar, A.T. Friberg, and D. Flavin, J.Opt. Soc. Am. B, 23 (8) (2006) $1540-1543$

[12] N.V. Nikonorov, A.K. Przhevuskii, A.V. Chukarev, J. Non-Cryst. Solids 324 (2003), 92-108

[13] Le Ngoc Chung, Chu Thi Thu Ha, Nguyen Thu Trang, Pham Thu Nga, and Pham Van Hoi, J. Mater. Scie Es Engin B, 131 (2006) 27-31

[14] B. Dong, D.P. Liu, X.J.Wang, T. Yang, S.M. Miao, and C. R. Li, Appl. Phys. Lett. 90 (2007), 181117

[15] Pham Van Hoi, Chu Thi Thu Ha, and Hoang Quang Hung, App. Phys. Letters. 87(2005), 161110/1-3

[16] S.V. Sergeyev and B. Jaskorzynska, Phys. Rev. B 62 (23) (2000) 15628-15633

Received 27 October 2009. 